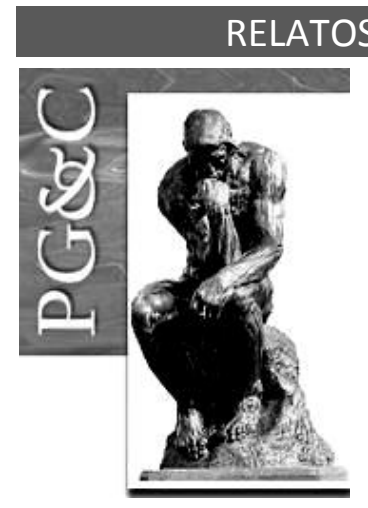

\title{
BARREIRAS AO COMPARTILHAMENTO DO CONHECIMENTO NAS ORGANIZAÇÕES
}

\author{
Lucimara Wons \\ Especialista em Planejamento e Gestão Estratégica pelo \\ Centro Universitário Internacional, Brasil. \\ E-mail: luci@wons.com.br \\ Rosilane de Oliveira Castro de Souza \\ Especialista em Finanças e Desenvolvimento Empresarial pelo \\ Centro Universitário Internacional, Brasil. \\ E-mail: rosilanecastro82@gmail.com \\ Helena de Fátima Nunes Silva \\ Doutora em Engenharia de Produção pela Universidade Federal de Santa \\ Catarina, Brasil. Professora da Universidade Federal do Paraná, Brasil. \\ E-mail: helenanuness@gmail.com \\ Flávia Roberta Fernandes \\ Mestre em Ciência, Gestão e Tecnologia da Informação pela \\ Universidade Federal do Paraná, Brasil. \\ E-mail: flaroberta@gmail.com
}

\begin{abstract}
Resumo
O compartilhamento de conhecimento é considerado um fator organizacional para manter as organizações competitivas no mercado, da mesma forma que um instrumento para promover o crescimento, agregar valor individual e institucional. Entretanto ao longo deste processo identificam-se fatores nas esferas individual, organizacional e tecnológico que impedem e dificultam a interação dos indivíduos, assim como a disponibilização e partilha deste conhecimento. 0 estudo tem por objetivo identificar os fatores que dificultam ou impedem o compartilhamento do conhecimento nas organizações, a partir de um levantamento bibliográfico na base de dados Library, Information Science \& Technology Abstracts (EBSCO). Foram recuperados doze artigos e as barreiras identificadas foram organizadas e sistematizadas em três categorias: barreira individual, barreira organizacional e barreira tecnológica. Os resultados demonstram que as barreiras apontadas com maior incidência nos artigos são na barreira individual: a falta de tempo, confiança, mentalidade individualista e falta de profundidade nos relacionamentos; na barreira organizacional: falta de infraestrutura e recursos, ausência de cultura organizacional saudável, idioma, limites/distância física e conflitos de comunicação; e na barreira tecnológica: dificuldade no manuseio de ferramentas e sistemas e dificuldades em integrar e modificar os sistemas baseados em tecnologia. Observa-se a necessidade de conscientização sobre os benefícios do compartilhamento do conhecimento e de uso de técnicas que propiciem e facilitem a criação e a gestão do conhecimento nas empresas.
\end{abstract}

Palavras-chave: Gestão do conhecimento. Compartilhamento do conhecimento. Barreiras ao compartilhamento do conhecimento.

\section{BARRIERS TO KNOWLEDGE SHARING IN ORGANIZATIONS}

\section{Abstract}

Knowledge sharing is considered an organizational factor to keep organizations competitive in the market, in the same way as an instrument to promote growth, to add individual and institutional value.

Perspectivas em Gestão \& Conhecimento, João Pessoa, v. 8, número especial, p. 86-101, out. 2018. DOI: http://dx.doi.org/10.21714/2236-417X2018v8nep86

http://periodicos.ufpb.br/ojs2/index.php/pgc. ISSN: 2236-417X. Publicação sob Licença (cc) EY-NC-ND 
However, throughout this process we identify factors in the individual, organizational and technological spheres that impede and hinder the interaction of individuals, as well as the availability and sharing of this knowledge. The study aims to identify the factors that hamper or impede the sharing of knowledge in organizations, from a bibliographic survey in the Library, Information Science \& Technology Abstracts (EBSCO) database. Twelve articles were retrieved and the barriers identified were organized and systematized into three categories: individual barrier; organizational barrier and technological barrier. The results show that the barriers pointed out with greater incidence in articles are in the individual barrier: lack of time, confidence, individualistic mentality and lack of depth in relationships; in the organizational barrier: lack of infrastructure and resources, absence of healthy organizational culture, language, physical limits / distance and communication conflicts; and the technological barrier: difficulty in handling tools and systems and difficulties in integrating and modifying technology-based systems. There is a need to raise awareness about the benefits of knowledge sharing and the use of techniques that facilitate and facilitate the creation and management of knowledge in companies.

Keywords: Knowledge Management. Knowledge sharing. Barriers to knowledge sharing.

\section{INTRODUÇÃO}

O mercado global competitivo evidencia a necessidade das organizações em perceber as mudanças que ocorrem externa e internamente, bem como estarem dispostas a adequar suas estratégias de gestão e forma de atuação (SANTIAGO, 2004). Segundo Santiago (2004), a maior mudança à qual as empresas têm que se submeter para se diferenciarem neste ambiente, diz respeito à rapidez com que suas atividades devem ser executadas e aprimoradas. Da mesma forma, é necessário levar em consideração que o valor dos produtos e serviços desenvolvidos está relacionado com inteligência, tecnologia e a inovação incorporados em seus processos (SANTIAGO, 2004). Segundo os autores Nonaka e Takeuchi (2008), o cerne para a inovação é a produção de conhecimento.

A produção do conhecimento e sua contribuição organizacional recebem uma maior atenção a partir dos anos 90, com o deslocamento da sociedade baseada na produção de bens/produtos para a sociedade do conhecimento (GIDDENS, 1991). O conhecimento "passa a ser o motor da economia e se transforma na principal forma de produção" (SCHLESINGER et al. 2008 , p. 5), assim como torna-se o único ativo que realmente diferencia e destaca competitivamente, as organizações (ASRAR-UL-HAQ; ANWAR, 2016; NONAKA; TAKEUCHI, 2003), visto sua superioridade diante dos demais ativos físicos ou financeiros (SANTIAGO, 2004).

Diante deste cenário de valorização do conhecimento e do desafio das organizações em identificar, produzir e compartilhar o conhecimento existente entre os membros (HONG, SUH, KOO, 2011; CHOO, 2006), a gestão do conhecimento operacionaliza processos para que os indivíduos acessem o conhecimento quando necessário, com o objetivo de aumentar o desempenho humano e organizacional (TERRA, 2001).

No processo da gestão do conhecimento organizacional, uma das etapas que contribui para o desempenho humano e institucional, influenciando o sucesso das empresas, envolve o compartilhamento do conhecimento, visto que este facilita a extração dos benefícios dos seus recursos existentes (KUKKO, 2013). Segundo Asrar-UI_Haq e Anwar (2016, p. 2) "entre outros processos da gestão do conhecimento, o compartilhamento de conhecimento foi considerado o mais vital". Além de ser "considerado um mecanismo de suporte crítico para a conclusão bem sucedida de atividades intensivas em conhecimento (ZAHEDI; SHAHIN; BABAR, 2016, p. 1012). No entanto, este compartilhamento de conhecimento é um desafio, pois os membros de uma organização podem ter diferentes perspectivas sobre o que possa ser importante para o negócio da organização, da mesma forma "por integrar múltiplos fatores que devem estar

Perspectivas em Gestão \& Conhecimento, João Pessoa, v. 8, número especial, p. 86-101, out. 2018. 
alinhados e serem administrados sistemicamente em iniciativas bem sucedidas de gestão do conhecimento" (WILBERT et al, 2014, p. 13).

A configuração e o tamanho da organização influenciam nas formas, no planejamento e nos meios do compartilhamento de conhecimento (ZAHEDI; SHAHIN; BABAR, 2016). Runte (2016) afirma que o processo de implantação de um sistema de Gestão de Conhecimento envolve muitos desafios para as organizações que precisam passar pelo rompimento de hábitos, crenças e valores, até então incorporados, e que refletem indiretamente na sua cultura organizacional e gestão. Com isso, é possível observar o surgimento de algumas barreiras que impedem o compartilhamento do conhecimento (ASRAR-UL HAQ; ANWAR, 2016).

Tendo em vista a importância da Gestão do Conhecimento e o crescente interesse no processo de compartilhamento de conhecimento nas organizações (WILBERT et al, 2014), da mesma forma que no âmbito acadêmico e nas instituições de pesquisas, optou-se por explorar o assunto a partir de um levantamento das barreiras que impedem o compartilhamento do conhecimento nas organizações, uma vez que, como pontuado por Wilbert et al (2014, p. 13) "o compartilhamento do conhecimento envolve não só a identificação e disponibilização de técnicas, ferramentas e tecnologias comumente recomendadas para esta etapa específica, como também a cultura favorável, os fatores estruturais, motivacionais e humanos". Diante disso, busca-se compreender os processos da gestão do conhecimento, especificamente o compartilhamento do conhecimento e os fatores que o limitam e que podem ocasionar falhas ou promover dificuldade no seu gerenciamento. Para tanto, a presente pesquisa tem como objetivo identificar estudos que apontem os fatores que dificultam ou impedem 0 compartilhamento do conhecimento nas organizações.

\section{REFERENCIAL TEÓRICO}

Alvarenga Neto (2008) afirma que, com o intuito de se manterem competitivas no mercado, as organizações migraram de uma abordagem gerencial econômica para a chamada Era do Conhecimento, cujo paradigma é sustentado pela informação, conhecimento e inovação. Da mesma forma, que diante de um mercado cada vez mais instável e dinâmico, as organizações precisam administrar as informações que lhes afetam como forma de adaptação, seja no seu ambiente interno ou nos seus relacionamentos externos (CHOO, 2006). Segundo Choo (2006), "uma organização do conhecimento é aquela que, por meio de uma estrutura conceitual, utiliza e cria informações estrategicamente para manter seu crescimento e sua capacidade de adaptação" (CHOO, 2006, p. 27).

Neste processo de adaptação e mudanças organizacionais, a Gestão de Conhecimento é um conjunto de processos que visam à criação, armazenamento, disseminação e utilização do conhecimento, alinhados com os objetivos da organização, considerando as fontes internas e externas (OLIVEIRA, et al., 2011), do mesmo modo, que possibilita a utilização das melhores informações e conhecimentos disponíveis, com a finalidade de se tomar decisões assertivas para obtenção dos objetivos e sucesso no mercado (ALVARENGA NETO, 2008). Davenport e Prusak (1998) apontam que a gestão de conhecimento nas organizações deve manter o foco no conhecimento e atender aos seguintes objetivos: (i) criação de um repertório de conhecimento, que seja constituído com o conhecimento externo, conhecimento interno estruturado e informal; (ii) facilitação do acesso ao conhecimento através da identificação das "páginas amarelas" e estabelecimento de rede de contatos com especialistas e criação de gatekeepers; (iii) desenvolvimento de um ambiente e uma cultura organizacional que estimulem a criação, transferência e o uso do conhecimento; e (iv) gerenciamento do conhecimento como recurso mensurável.

Perspectivas em Gestão \& Conhecimento, João Pessoa, v. 8, número especial, p. 86-101, out. 2018. 
Da mesma forma que a organização deve atentar para a criação de um repertório, a facilitação do acesso ao conhecimento, o desenvolvimento de um ambiente e cultura de promoção e gerenciamento do conhecimento (DAVENPORT; PRUSAK, 1998) faz-se necessário a identificação dos tipos de conhecimento, que como apontado por Nonaka e Takeuchi (2008), dividem-se em explícito e tácito. O conhecimento explícito pode ser expresso em palavras, números, sons, e compartilhado na forma de dados, fórmulas científicas e recursos visuais, enquanto o conhecimento tácito não é facilmente explicável, pois está associado à intenção, valores e crenças dos indivíduos, criando dificuldades para sua visibilidade, explicação e formalização. O conhecimento tácito abrange os conhecimentos cognitivos e técnicos, modelos mentais, crenças e perspectivas pessoais dos indivíduos, sendo estes fatores que exercem influências na maneira em que cada indivíduo percebe o mundo em sua volta (NONAKA; TAKEUCHI, 1997).

Segundo Nonaka e Takeuchi $(1997,2008)$ o conhecimento é criado e compartilhado por meio de um processo de interação social entre o conhecimento tácito e explícito, sendo composto por quatro modos de conversão de conhecimento, sendo: (i) socialização (tácito em tácito); (ii) externalização (tácito em explícito); (iii) combinação (explícito em explícito); e (iv) internalização (explícito em explícito). A conversão do conhecimento ocorre em um processo cíclico em espiral, denominado SECl. Segundo Nonaka, Toyama e Konno (2000, p. 14), um fator que contribui para o processo de conversão do conhecimento envolve o ambiente, denominado pelos autores de "Ba". O Ba refere-se ao ambiente em que o indivíduo absorve e interpreta a informação tornando-a conhecimento, podendo este ser um local físico, virtual ou mental (NONAKA; TOYAMA; KONNO, 2000).

A criação de ambientes como o Ba (NONAKA; TOYAMA; KONNO, 2000), que promovam a interação dos indivíduos nas organizações, coopera para que o conhecimento seja compartilhado (HOOFF; HUYSMAN, 2009), internalizado e aplicado com o intuito de gerar novos conhecimentos (SHLESINGER et al, 2008). De acordo com Di Chiara, Alcará e Tomael (2010, p. 106) "o conhecimento não é estimulado por meio da imposição de estruturas e ferramentas, mas por sua rica interação social". Da mesma forma que, para que este percorra o ambiente organizacional e transforme-se de um conhecimento tácito, presente nos indivíduos e na organização, em conhecimento explícito, as relações estabelecidas são consideradas um fator primordial, "permitindo à organização obter, por meio deste conhecimento [explícito], o desempenho superior tão esperado" (QUANDT; PEREIRA JR; VALE, 2014, destaque das autoras). Segundo Hooff e Huysman (2009) a interação dos indivíduos proporciona a construção e o compartilhamento do conhecimento, visto que este é um processo que ocorre de forma conjunta, atribuindo significado aos fatos e situações. No entanto, o grande desafio para as organizações consiste em fazer com que o conhecimento seja compartilhado entre todos os membros da organização (NONAKA; TAKEUCHI, 1997), assim como gerenciado, a fim de promover sua permanência em um mercado de crescente competitividade (ASRAR-UL-HAQ; ANWAR, 2016).

\subsection{Barreiras ao compartilhamento do conhecimento}

O processo de criação do conhecimento envolve o compartilhamento de conhecimento (NONAKA; TAKEUCHI, 1997) e este, por sua vez, exerce um papel indispensável para a inovação nas organizações, uma vez que o conhecimento compartilhado contribui para novas ideias, implementação de processos, produtos e serviços (ORDAZ; CRUZ; GINEL, 2009). Neste processo, deve-se considerar que o conhecimento reside nos membros pertencentes à organização (HONG; SUH; KOO, 2011) e depende da solicitude em transmiti-lo, para que os demais indivíduos possam se beneficiar e utilizá-lo (ALCARÁ et al., 2009). Entretanto, os indivíduos possuem diferentes pontos de vista diante do que é importante compartilhar e

Perspectivas em Gestão \& Conhecimento, João Pessoa, v. 8, número especial, p. 86-101, out. 2018. 
outros fatores podem interferir no compartilhamento do conhecimento (HONG; SUH; KOO, 2011), como crenças, valores e costumes individuais que integram a cultura organizacional (DI CHIARA; ALCARA; TOMAEL, 2010). Portanto, a produção ou criação de conhecimento, numa perspectiva diferente daquela voltada apenas à aquisição de conhecimento, depende da socialização e interação das pessoas para que os conhecimentos já produzidos possam ser transformados em novos conhecimentos (NONAKA; TAKEUCHI, 1997; MIRANDA, 1997).

Os aspectos facilitadores e os impedimentos ao conhecimento, em uma organização, estão relacionados com a cultura dos colaboradores. Para tanto, "é essencial que os líderes disponibilizem práticas compatíveis ao compartilhamento do conhecimento, para que os colaboradores aceitem as mudanças de forma positiva, tornando a organização mais competitiva" (PEREIRA; BARBOSA, 2018, p. 13). De acordo com Riege (2005), a identificação e o reconhecimento das barreiras de compartilhamento de conhecimento desempenham um papel importante no sucesso de uma estratégia de Gestão de Conhecimento e pode ou não, fazer parte da cultura de uma organização. $O$ alinhamento entre a gestão do conhecimento e a estratégia organizacional é um ponto crucial visto que para o atingimento, o compartilhamento é tido como uma atividade isolada ou separada (RIEGE, 2005). Segundo Rizzon et al. (2016) o compartilhamento de conhecimento nas organizações está relacionado à atitude dos indivíduos, aos contextos organizacionais e culturais, seja no nível local ou macroambiente, ao acesso e disseminação dos recursos tecnológicos de informação e comunicação, assim como "a cultura favorável, os fatores estruturais, motivacionais e humanos" (WILBERT, et al, 2014, p. 13). Diante disso, podem ser consideradas barreiras segundo as esferas individual, organizacional e tecnológica, sendo:

(1) individual: falta de habilidade de comunicação e redes sociais, diferenças na cultura nacional, excesso de ênfase nos status de posição (RIEGE, 2005), falta de tempo (RIEGE, 2005; RIZZON et al, 2016), receio de constrangimento e incertezas (DISTERER, 2001), perda de poder (DISTERER, 2001; RIZZON et al, 2016), resistência interna, motivação e a percepção de lacunas no conhecimento (HONG; SUH; KOO, 2011), dificuldade em perceber os benefícios, mentalidade individualista, falta de profundidade nos relacionamentos e o receio de serem superados pelos colegas, além da falta de reciprocidade na partilha do conhecimento (RIZZON et al, 2016) e confiança (ALCARÁ, et al, 2009); HONG; SUH; KOO, 2011; RIEGE, 2005).

(2) organizacional: ligadas à viabilidade econômica, à falta de infraestrutura e recursos, à acessibilidade nos espaços de reuniões formais e informais e ao meio ambiente (RIEGE, 2005), o idioma, a prevenção de conflitos, burocracia, distância (HONG; SUH; KOO, 2011), ausência de uma cultura organizacional saudável, ausência de incentivos ou recompensas a quem compartilha seu conhecimento, linguagem, burocracia, limites físicos que impõem distância entre os trabalhadores, hierárquica e conflitos de comunicação foram identificados como barreiras ao compartilhamento de conhecimento organizacionais (RIZZON et al., 2016).

(3) tecnológica: as tecnologias da informação apoiam e facilitam a troca e o compartilhamento de conhecimento (HOOFF; HUYSMAN, 2009) e as barreiras identificadas correlacionam-se com fatores que envolvem a dificuldade no manuseio de ferramentas e sistemas, visto a incompatibilidade com a real necessidade do usuário, dificuldades em integrar e modificar os sistemas baseados em tecnologia (RIEGE, 2005), a dificuldade ao acesso às ferramentas, falta de consciência da existência da partilha de conhecimento nas plataformas, ausência de treinamento e estímulos para a utilização das mesmas, insuficiência de competência em tecnologia de informação e instalações inadequadas (RIZZON et al., 2016).

Os autores Pupulim, Fialho e Varvális (2017), apresentam um levantamento bibliográfico quanto às barreiras identificadas nos processos de gestão do conhecimento e analisando os itens, destacam-se os que podem ser relacionados aos fatores individuais, organizacionais e tecnológicos ao compartilhamento do conhecimento (Quadro 1).

Perspectivas em Gestão \& Conhecimento, João Pessoa, v. 8, número especial, p. 86-101, out. 2018. 
Quadro 1 - Barreiras à Gestão do Conhecimento em comparação com o Compartilhamento de Conhecimento

\begin{tabular}{|c|c|c|c|}
\hline $\begin{array}{l}\text { Barreiras à } \\
\text { Gestão do } \\
\text { Conhecimento }\end{array}$ & Autores citados & Esfera & $\begin{array}{c}\text { Relação com o compartilhamento } \\
\text { de conhecimento. }\end{array}$ \\
\hline $\begin{array}{l}\text { Diversidade } \\
\text { cultural dos } \\
\text { integrantes da } \\
\text { equipe }\end{array}$ & $\begin{array}{c}\text { Thomas, 1996; Cormican; } \\
\text { Dooley, 2007; Obikunle, 2002; } \\
\text { Rodrigues, 2010; Stare, 2011; } \\
\text { Ortiz, 2014. }\end{array}$ & Individual & $\begin{array}{l}\text { diferença na cultura nacional } \\
\text { (RIEGE, 2005) }\end{array}$ \\
\hline $\begin{array}{l}\text { Falta de } \\
\text { motivação } \\
\text { pessoal }\end{array}$ & $\begin{array}{l}\text { Dyer; Nobeoka,2000; Krogh; } \\
\text { Ichijo; Nonaka, 2001; Ipe, } \\
\text { 2003; Huysman; Wit, 2004; } \\
\text { Cormican; Dooley, 2007; Alves; } \\
\text { Barbosa, 2010; Barros et al, } \\
\text { 2010. }\end{array}$ & Individual & $\begin{array}{c}\text { motivação (HONG; SUH; KOO, } \\
\text { 2011) }\end{array}$ \\
\hline $\begin{array}{l}\text { Falta de } \\
\text { confiança } \\
\text { nos pares }\end{array}$ & $\begin{array}{l}\text { Goman (2002); Cormican; } \\
\text { Dooley, 2007; Alves; Barbosa, } \\
\text { 2010; Barros et al, } 2010 .\end{array}$ & Individual & $\begin{array}{l}\text { confiança (ALCARÁ, et al, 2009); } \\
\text { HONG; SUH; KOO, 2011; RIEGE, } \\
\text { 2005). }\end{array}$ \\
\hline $\begin{array}{l}\text { Resistência a } \\
\text { mudanças }\end{array}$ & $\begin{array}{l}\text { Nonaka; Takeuchi, 1997; } \\
\text { Szulanski, 2000; Davenport; } \\
\text { Prusak, 2003; Barros et al, } \\
\text { 2010. }\end{array}$ & Individual & $\begin{array}{l}\text { resistência interna (HONG; SUH; } \\
\text { KOO, 2011). }\end{array}$ \\
\hline $\begin{array}{l}\text { Problemas de } \\
\text { comunicação }\end{array}$ & $\begin{array}{c}\text { Moscovici, 1999; Robbins, } \\
\text { 2002; Kunsch, 2003; Cormican; } \\
\text { Doolev, } 2007 .\end{array}$ & Individual & $\begin{array}{c}\text { falta de habilidade de } \\
\text { comunicação (RIEGE, 2005). }\end{array}$ \\
\hline $\begin{array}{l}\text { Ausência de } \\
\text { ferramentas } \\
\text { adequadas }\end{array}$ & $\begin{array}{c}\text { Mcafee, 2006; Cormican; } \\
\text { Dooley, 2007; Alves; Barbosa, } \\
2010 .\end{array}$ & $\begin{array}{l}\text { Tecnoló- } \\
\text { gica }\end{array}$ & $\begin{array}{l}\text { dificuldade no manuseio de } \\
\text { ferramentas e sistemas (RIEGE, } \\
\text { 2005). }\end{array}$ \\
\hline $\begin{array}{l}\text { Barreiras à } \\
\quad \text { G. } C\end{array}$ & Autores citados & Esfera & $\begin{array}{l}\text { Relação com o compartilhamento } \\
\text { de conherimento. }\end{array}$ \\
\hline $\begin{array}{l}\text { Ausência } \\
\text { orientação ao } \\
\text { compartilha- } \\
\text { mentn }\end{array}$ & Wilson,1997. & $\begin{array}{l}\text { Organiza- } \\
\text { cional }\end{array}$ & $\begin{array}{l}\text { ausência de incentivos ou } \\
\text { recompensas a quem compartilha } \\
\text { seu conhecimento (RIZZON et al., } \\
\text { ग016) }\end{array}$ \\
\hline $\begin{array}{l}\text { Aspectos } \\
\text { econômicos } \\
\text { organizacionais }\end{array}$ & $\begin{array}{l}\text { Wilson, 1997; Mcafee, 2006; } \\
\text { Ageron; Gunasekaran; } \\
\text { Spalanzani, 2011; Luthra et al, } \\
\text { ग011. }\end{array}$ & \multirow[t]{2}{*}{$\begin{array}{l}\text { Organiza- } \\
\text { cional }\end{array}$} & \multirow[t]{2}{*}{$\begin{array}{l}\text { ligadas à viabilidade econômica, à } \\
\text { falta de infraestrutura e recursos } \\
\text { (RIEGE, 2005). }\end{array}$} \\
\hline Custos & Luthra et al, 2011. & & \\
\hline Língua & $\begin{array}{l}\text { Krogh; Ichijo; Nonaka,2001; } \\
\text { Machado; Desideri, 2002; } \\
\text { Robbins, 2002; Kunsch, 2003; } \\
\text { Barros et al. } 2010 .\end{array}$ & $\begin{array}{l}\text { Organiza- } \\
\text { cional }\end{array}$ & $\begin{array}{c}\text { o idioma (HONG, SUH, KOO, 2011) } \\
\text { e linguagem } \\
\text { (RIZZON et al., 2016). }\end{array}$ \\
\hline
\end{tabular}

Perspectivas em Gestão \& Conhecimento, João Pessoa, v. 8, número especial, p. 86-101, out. 2018. 


\begin{tabular}{|c|c|c|c|}
\hline $\begin{array}{l}\text { Barreiras à } \\
\text { Gestão do } \\
\text { Conhecimento }\end{array}$ & Autores citados & Esfera & $\begin{array}{l}\text { Relação com o compartilhamento } \\
\text { de conhecimento. }\end{array}$ \\
\hline $\begin{array}{c}\text { Aspectos } \\
\text { geográficos }\end{array}$ & $\begin{array}{l}\text { Wilson,1997; Machado; } \\
\text { Desideri, } 2002 .\end{array}$ & $\begin{array}{l}\text { Organiza- } \\
\text { cional }\end{array}$ & $\begin{array}{l}\text { limites físicos que impõem } \\
\text { distância entre os trabalhadores } \\
\text { (RIZZON et al. 2016). }\end{array}$ \\
\hline $\begin{array}{c}\text { Cultura } \\
\text { organizacional }\end{array}$ & $\begin{array}{l}\text { Baccarelli, 1999; Leuch; } \\
\text { Carvalho; Kovaleski, 2005; } \\
\text { Barbosa: Senúlveda: Costa. }\end{array}$ & $\begin{array}{l}\text { Organiza- } \\
\text { cional }\end{array}$ & $\begin{array}{c}\text { ausência de uma cultura } \\
\text { organizacional saudável (RIZZON } \\
\text { et al. 2016). }\end{array}$ \\
\hline $\begin{array}{c}\text { Barreiras } \\
\text { tecnológicas }\end{array}$ & Schwartz et al, 1999. & $\begin{array}{l}\text { Tecnoló- } \\
\text { gica }\end{array}$ & $\begin{array}{c}\text { dificuldades com: acesso e } \\
\text { manuseio de } \\
\text { ferramentas/sistemas, (RIEGE), } \\
\text { 2005), conscientização da partilha } \\
\text { de conhecimento nas plataformas, } \\
\text { ausência de } \\
\text { treinamento/estímulos para a } \\
\text { utilização, competência no } \\
\text { manuseio (RIZZON et al., 2016). }\end{array}$ \\
\hline
\end{tabular}

Fonte: Adaptado de Pupulim, Fialho e Varvakis (2017, p. 225)

Conforme levantamento realizado por Pupulim, Fialho e Varvakis (2017), podem ser destacadas as barreiras à G.C que são, da mesma forma, identificadas no processo de compartilhamento de conhecimento: (1) individual - falta de habilidade de comunicação, diferenças na cultura nacional (RIEGE, 2005), resistência interna, motivação (HONG; SUH; KOO, 2011) e confiança (ALCARÁ, et al, 2009; HONG; SUH; KOO, 2011; RIEGE, 2005); (2) organizacional - ausência de uma cultura organizacional saudável, ausência de incentivos ou recompensas a quem compartilha seu conhecimento, linguagem, limites físicos que impõem distância entre os trabalhadores (RIZZON et al, 2016); e (3) dificuldade no manuseio de ferramentas e sistemas (RIEGE, 2005), dificuldade ao acesso às ferramentas, falta de consciência da existência da partilha de conhecimento nas plataformas, ausência de treinamento e estímulos para a utilização das mesmas, insuficiência de competência em tecnologia de informação e instalações inadequadas (RIZZON et al., 2016).

Segundo Alcará, et al. (2009), o compartilhamento de conhecimento sofre influência de fatores internos e externos e sua identificação e análise fornece informações às organizações para que desenvolvam ações, estimulem os indivíduos e promover ambientes para a interação e o estabelecimento de confiança entre os membros. Para Maronato e Stankowitz (2017, p. 123) "existe uma relação de interdependência entre confiança e a disposição para o compartilhamento de conhecimento entre os indivíduos na organização". Da mesma forma que apontado por Quandt, Pereira Jr. e Vale (2014, p. 14) que a confiança entre os indivíduos "permite atitudes positivas a respeito do compartilhamento do conhecimento, e como o valor percebido pelo compartilhamento do conhecimento reforça os relacionamentos dentro das organizações". Da mesma forma, deve-se existir a promoção de condições e um contexto que favoreça o compartilhamento (ORDAZ; CRUZ; GINEL, 2009), assim como os indivíduos compreendam os benefícios pessoais e profissionais que o compartilhamento de conhecimento pode gerar (PUPULIM; FIALHO; VARVAKIS, 2017).

O levantamento realizado por Santos, Oliveira e Luciano (2017), aponta que com a disseminação das redes sociais no final da década de 2000, onde o compartilhamento de conhecimento também passou a ser feito por comunidades virtuais, as organizações estão vivendo um dilema entre compartilhar e proteger o seu conhecimento, visto que, o

Perspectivas em Gestão \& Conhecimento, João Pessoa, v. 8, número especial, p. 86-101, out. 2018. 
compartilhamento de conhecimento por meio de mídias pode ser tanto uma oportunidade como um risco, devido à abrangência e velocidade que pode alcançar. O compartilhamento interorganizacional do conhecimento tácito pode representar um risco para as organizações no que diz respeito à manutenção de vantagem competitiva (SANTOS, OLIVEIRA; LUCIANO, 2017).

\section{METODOLOGIA}

Neste estudo, de abordagem qualitativa e quantitativa (mista), realizou-se pesquisa bibliográfica, pautada em material científico já elaborado (GIL, 2008), com o objetivo de identificar as barreiras ao compartilhamento do conhecimento nas organizações.

Segundo Wilbert et al (2014, p. 3-4), desde a identificação do primeiro artigo com o termo "knowledge sharing", tem sido crescente a identificação da abordagem no tema, em bases de dados de periódicos internacionais. Por este motivo, o processo de coleta de dados baseou-se na consulta à plataforma da base de dados Library, Information Science \& Technology Abstracts with Full Text - LISTA (EBSCO). Optou-se pela referida base, pelo alinhamento ao tema proposto e a área Ciência da Informação, além de retornar a maior quantidade de publicações registradas.

A estratégia de busca aplicada na base de dados foi formulada com os termos: barreiras ao compartilhamento do conhecimento (barriers), obstáculos ao compartilhamento do conhecimento (obstacles) e empresas (company, business, organizations, firms, enterprises), por meio da expressão ((knowledge sharing barrier* OR knowledge sharing obstacle*) AND (compan* OR business OR organization* OR firm* OR enterprise*)). Recuperou-se um total de 486 artigos. Em seguida, definiu-se um filtro quanto ao período, sendo entre 2012 a 2017, a busca dos termos, aplicada aos títulos das publicações e a eliminação das duplicidades. Observou-se o total de 15 publicações, entretanto 3 estudos foram desconsiderados, visto a divergência quanto ao tema pesquisado e aos idiomas considerados, sendo inglês e português. Os 12 artigos restantes foram lidos integralmente para identificação das barreiras.

As barreiras ao compartilhamento do conhecimento, identificadas a partir do referencial teórico, nas esferas individual, organizacional e tecnológica, constituíram subcategorias para relacionar as barreiras apresentadas nos artigos recuperados, sendo:

a) subcategorias barreira individual: falta habilidade de comunicação; diferença cultural; receio de superação pelos colegas; ênfase no status/posição; perda de poder; receio de constrangimento; resistência interna; falta de tempo; motivação; identificação dos benefícios; mentalidade individualista; falta de profundidade nos relacionamentos; confiança; percepção de lacunas no conhecimento; e falta de reciprocidade.

b) subcategorias barreira organizacional: falta de infraestrutura e recursos; acessibilidade aos espaços formais e informais; idioma; prevenção de conflitos; ausência de cultura organizacional saudável; ausência de incentivos ou recompensas; linguagem; burocracia; limites/distância física; hierárquica; e conflitos de comunicação.

c) subcategorias barreira tecnológica: dificuldade no manuseio de ferramentas e sistemas; dificuldades em integrar e modificar os sistemas baseados em tecnologia; dificuldade ao acesso às ferramentas; falta de consciência da existência da partilha de conhecimento nas plataformas; ausência de treinamento e estímulos para a utilização das ferramentas; insuficiência de competência em tecnologia de informação; e instalações inadequadas.

Perspectivas em Gestão \& Conhecimento, João Pessoa, v. 8, número especial, p. 86-101, out. 2018. 


\section{RESULTADOS}

A partir da recuperação dos artigos, os dados foram analisados e organizados inicialmente a partir dos títulos, autores e as barreiras identificadas foram organizadas pelo escopo dos artigos, conforme apresentado na Figura 1:

Figura 1 - Barreiras identificadas a partir do escopo dos artigos

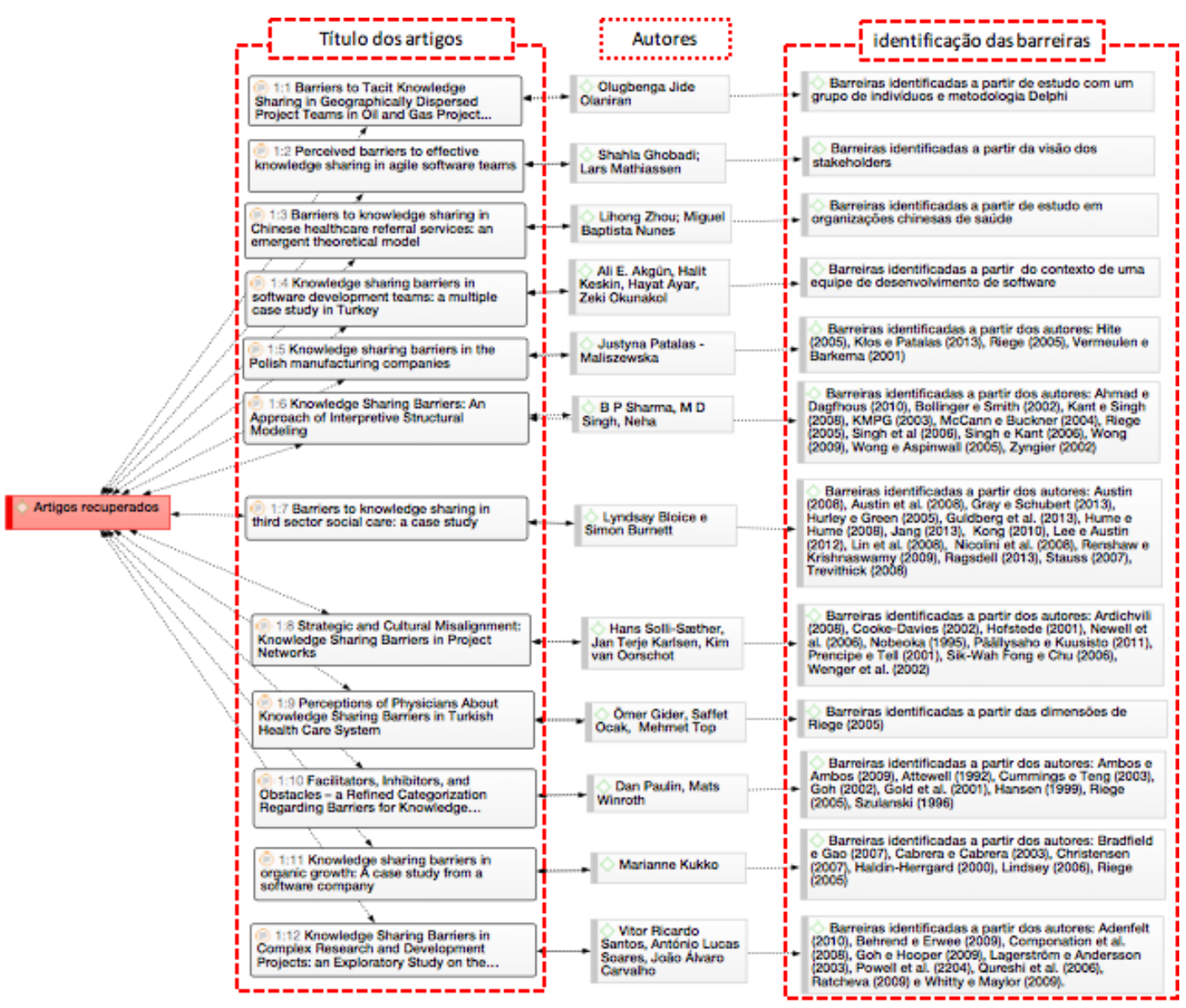

Fonte: As autoras com o auxílio do software Atlas.Ti (2018)

Observa-se na Figura 1 que oito artigos, sendo: (1) "Knowledge sharing barriers in the Polish manufacturing companies"; (2) "Knowledge sharing barriers: an approach of interpretive structural modeling"; (3) "Barriers to knowledge sharing in third sector social care: a case study"; (4) "Strategic and cultural misalignment: knowledge sharing barriers in project networks"; (5) "Perceptions of physicians about knowledge sharing barriers in Turkish health care system"; (6) "Facilitators, inhibitors, and obstacles - a refined categorization regarding barriers for knowledge transfer, sharing, and flow"; (7) "Knowledge sharing barriers in organic growth: a case study from a software company"; e (8) "Knowledge sharing barriers in complex research and development projects: an exploratory study on the perceptions of project manager", identificam as barreiras para o compartilhamento do conhecimento, pautados em um arcabouço teórico de autores que discutem a temática.

Com base na leitura dos artigos observou-se que as barreiras individuais, organizacionais e tecnológicas, com maior incidência nos estudos são aquelas, apresentadas conforme as subcategorias definidas na Figura 2:

Perspectivas em Gestão \& Conhecimento, João Pessoa, v. 8, número especial, p. 86-101, out. 2018. 
Figura 2 - Barreiras (individual, organizacional e tecnológica) com maior incidência nos artigos

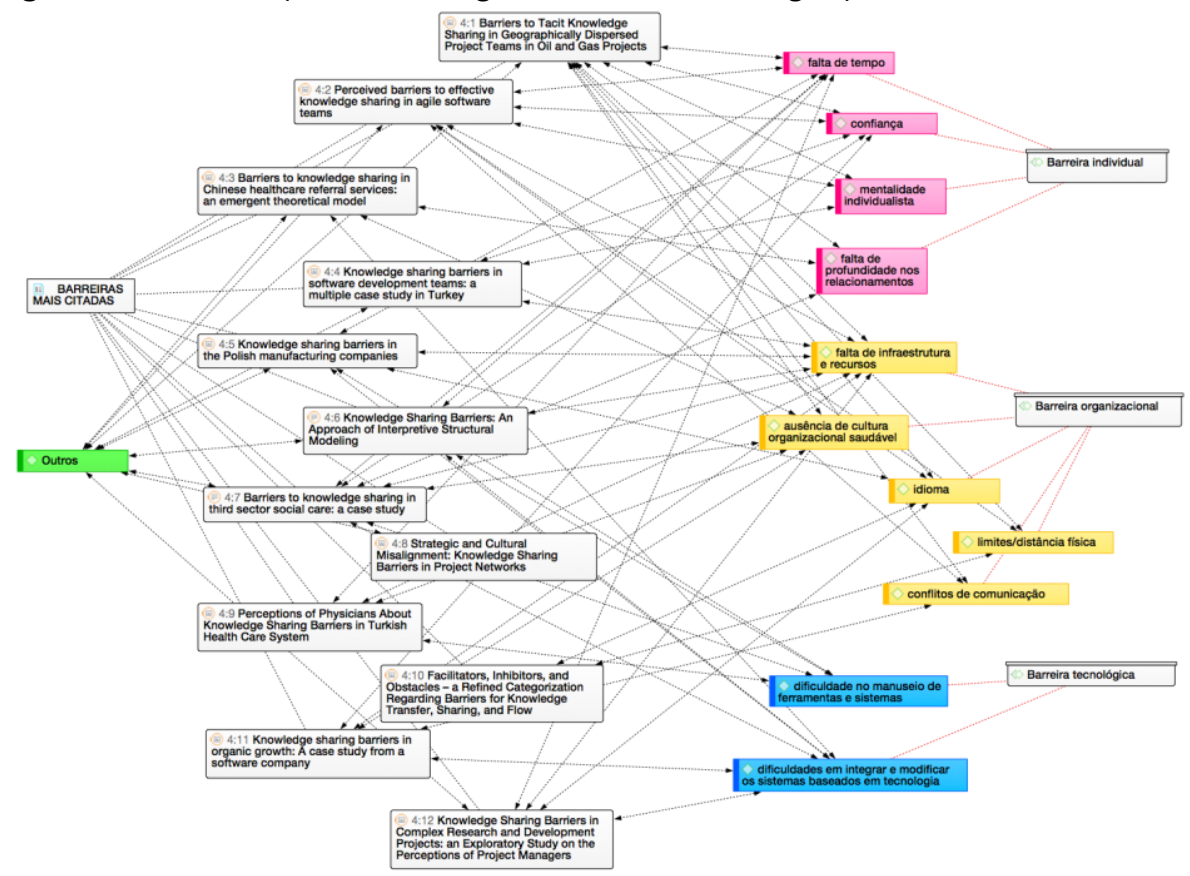

Fonte: As autoras com o auxílio do software Atlas.Ti (2018)

Observa-se na Figura 2, as barreiras identificadas com maior incidência em cada artigo, as quais foram agrupadas de acordo com as categorias, sendo: (a) barreira individual, a falta de tempo (7), confiança (5), mentalidade individualista (3) e falta de profundidade nos relacionamentos (3); (b) barreira organizacional, falta de infraestrutura e recursos (9), ausência de cultura organizacional saudável (5) idioma (5), limites/distância física (3) e conflitos de comunicação (3); e (c) barreira tecnológica, dificuldade no manuseio de ferramentas e sistemas (4) e dificuldades em integrar e modificar os sistemas baseados em tecnologia (6). Foram incluídas como "outras" as barreiras não citadas pelos autores do referencial teórico, englobando: o medo de colocar em perigo a segurança dos indivíduos, as diferenças culturais e a confiança envolvendo, indivíduos, equipes e empresas.

As Figura 3, Figura 4 e Figura 5, apresentam as demais barreiras identificadas de acordo com as categorias: (1) individual; (2) organizacional; e (3) tecnológica.

Figura 3 - Demais barreiras individuais identificadas, agrupadas de acordo com as subcategorias

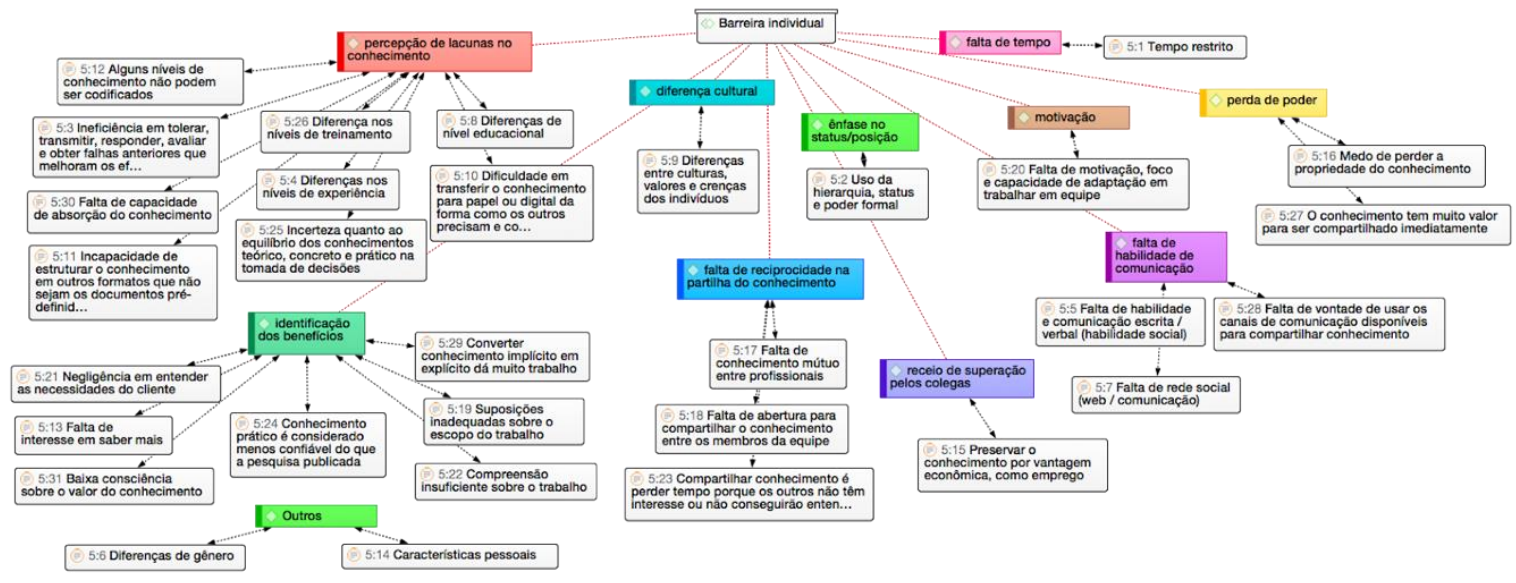

Fonte: As autoras com auxílio do software Atlas ti (2018)

Perspectivas em Gestão \& Conhecimento, João Pessoa, v. 8, número especial, p. 86-101, out. 2018. 
Conforme apresentado na Figura 3, as demais barreiras individuais, identificadas de acordo com as subcategorias definidas nos artigos recuperados foram: percepção de lacunas do conhecimento; identificação dos benefícios; diferença cultural; ênfase no status/posição; falta de tempo; motivação; perda de poder; falta de habilidade de comunicação; falta de reciprocidade; e falta de reciprocidade na partilha do conhecimento.

A Figura 4, apresenta as demais barreiras organizacionais identificadas, de acordo com as subcategorias.

Figura 4 - Demais barreiras organizacionais identificadas, de acordo com as subcategorias

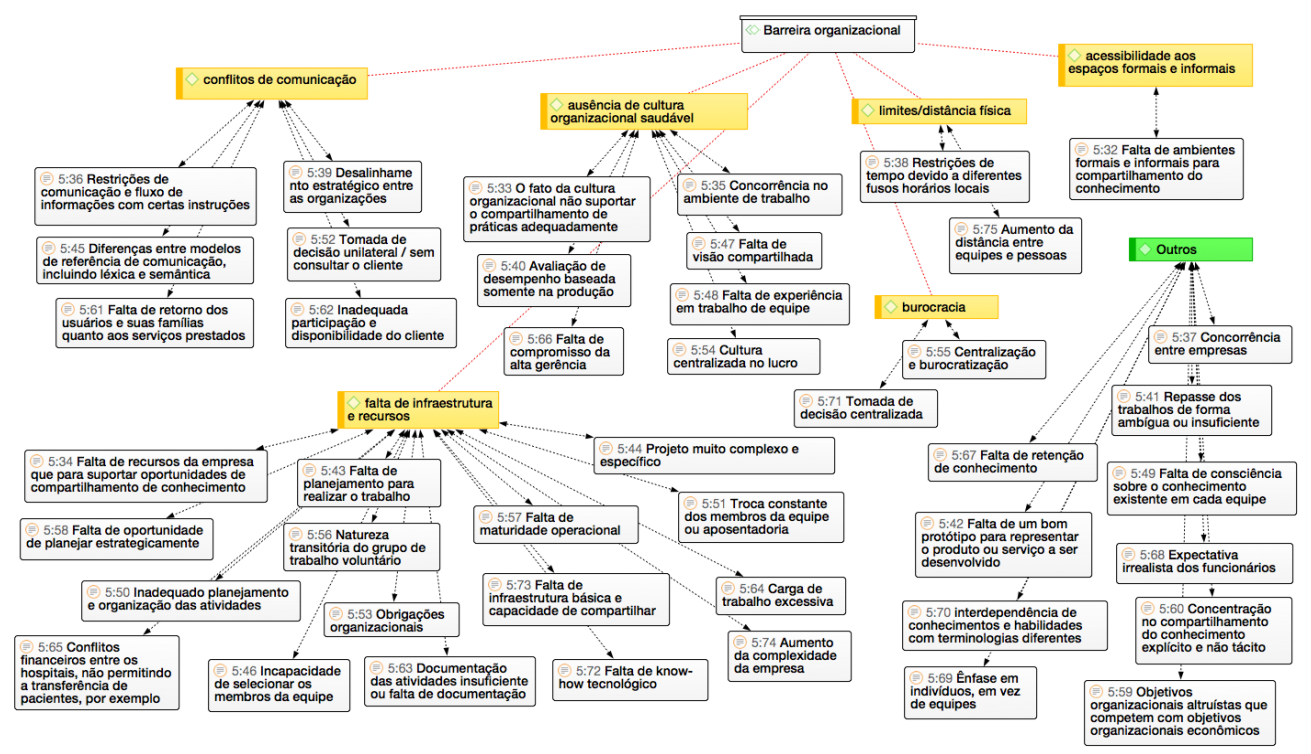

Fonte: As autoras com o auxílio do software Atlas.Ti (2018)

Observa-se na Figura 4, que as barreiras organizacionais, identificadas de acordo com subcategorias foram: conflitos de comunicação; ausência de cultura organizacional saudável; limites/distância física; acessibilidade aos espaços formais e informais; burocracia; e falta de infraestrutura e recursos.

Em relação às demais barreiras tecnológicas, foram identificadas as subcategorias, ausência de treinamento e estímulo para a utilização das ferramentas tecnológicas, conforme destacado na Figura 5.

Figura 5 - Demais barreiras tecnológicas identificadas, de acordo com as subcategorias

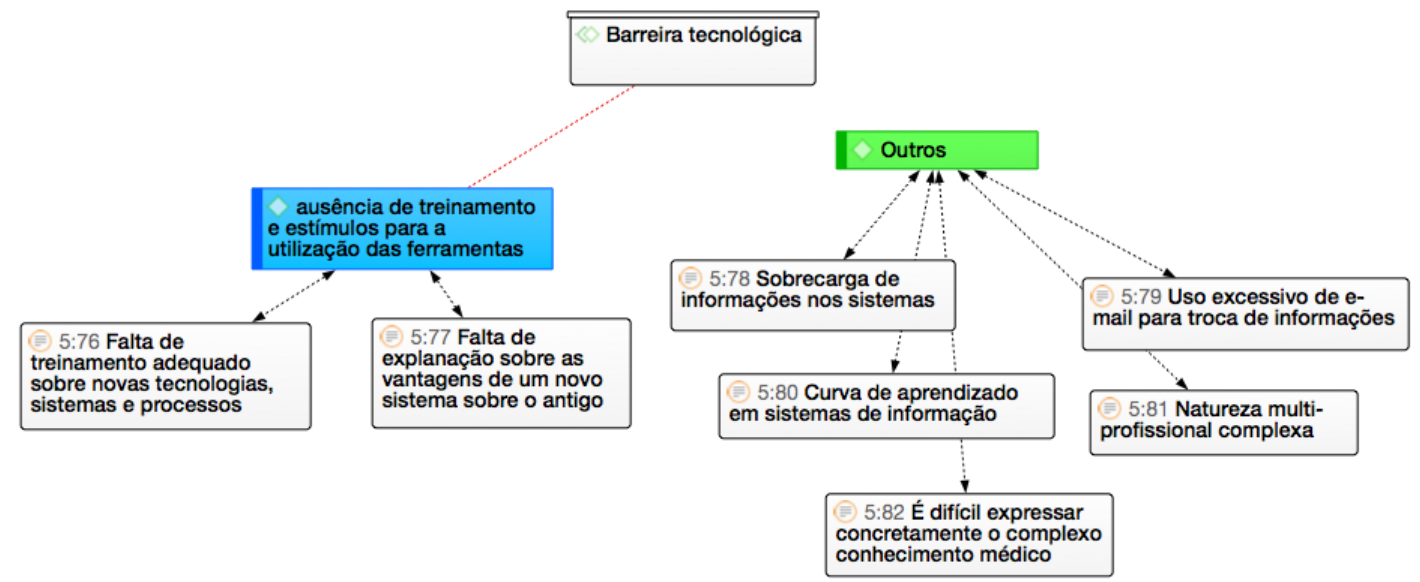

Fonte: As autoras com auxílio do software Atlas.Ti (2018)

Perspectivas em Gestão \& Conhecimento, João Pessoa, v. 8, número especial, p. 86-101, out. 2018. 
A partir dos artigos recuperados analisou-se a dispersão das publicações ao longo dos seis anos pesquisados, conforme apresentado no Gráfico 1.

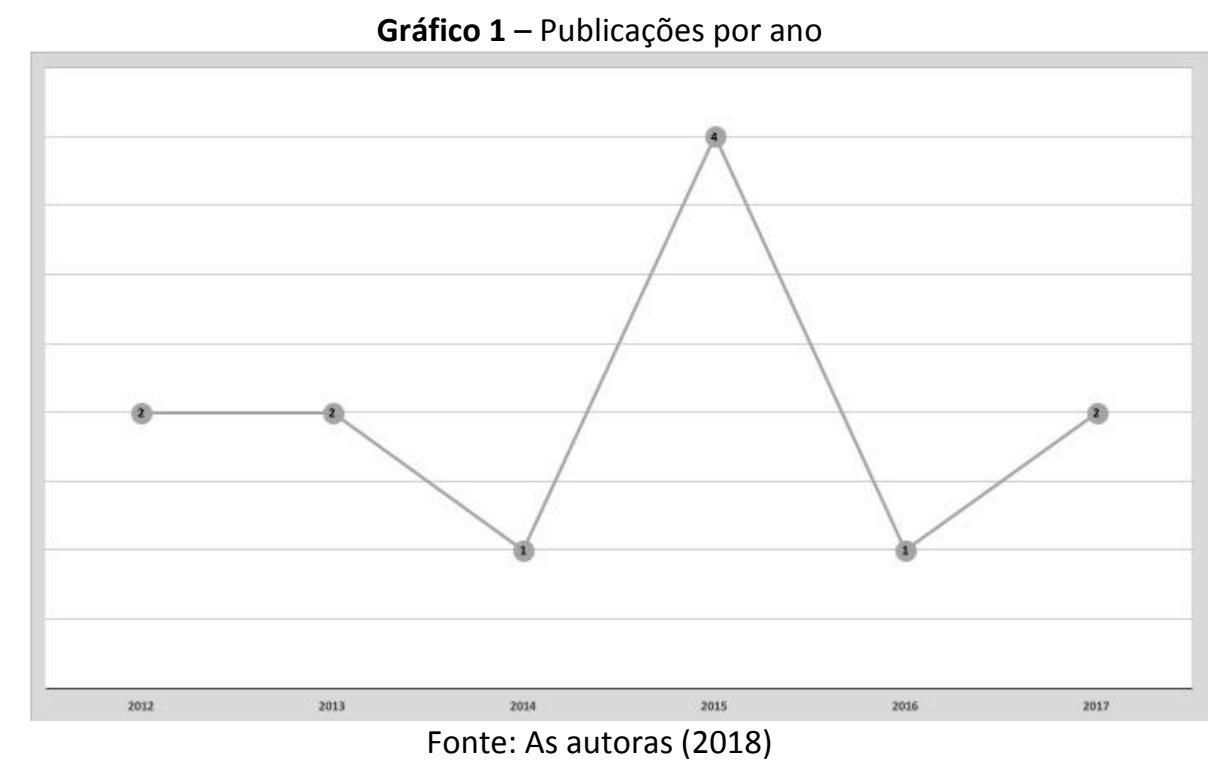

O ano de 2015 foi o mais produtivo com quatro publicações, seguidos dos anos 2012, 2013 e 2017, com duas publicações. As áreas que apresentaram publicações envolvendo as barreiras ao compartilhamento de conhecimento são: tecnologia da informação (4 artigos), construção civil ( 2 artigos), indústria ( 2 artigos), saúde ( 2 artigos), assistência social (1 artigo) e as organizações em geral (1 artigo). O continente com maior número de publicações é a Europa (Polônia, Escócia, Noruega, Suécia, Finlândia e Portugal), com $50 \%$ dos estudos seguido da Ásia (Turquia, China e Índia), com 25\% do total.

\section{CONSIDERAÇÕES FINAIS}

O conhecimento é considerado a base para o desenvolvimento das pessoas e das organizações e sua gestão, sendo necessária para o transcorrer das ações e interações individuais e institucional. Uma gestão eficaz do conhecimento permite que as empresas cresçam, gerando economia de recursos em todos os aspectos: financeiro, tempo e naturais. Por isso, a importância de conhecer as barreiras e fatores que dificultam seu compartilhamento. Para tanto, o presente artigo buscou compreender os processos da gestão do conhecimento, voltando-se para o compartilhamento do conhecimento e os fatores limitantes que podem promover ou dificultar o seu gerenciamento. Dentre as barreiras apresentadas, as com maior incidência nos artigos recuperados apontaram: falta de tempo, confiança, mentalidade individualista, falta de profundidade nos relacionamentos (barreira individual), falta de infraestrutura e recurso, ausência de cultura organizacional saudável, idioma, limites/distância física, conflitos de comunicação (barreira organizacional), dificuldade no manuseio de ferramentas e sistemas e dificuldades em integrar e modificar os sistemas baseados em tecnologia (barreira tecnológica).

As barreiras ao compartilhamento do conhecimento envolvem o indivíduo, a organização e o meio com o qual estes estabelecem relacionamentos. Uma vez identificadas as barreiras, torna-se possível promover ações individuais e organizacionais que estabeleçam os relacionamentos entre os membros de uma instituição, da mesma forma que construir um ambiente propício ao compartilhamento, seja este presencial ou suportado por tecnologias da

Perspectivas em Gestão \& Conhecimento, João Pessoa, v. 8, número especial, p. 86-101, out. 2018. 
informação. O compartilhamento de conhecimento (tanto aquele indicado no referencial teórico quanto aquele resultado da Revisão Sistemática da literatura) agrega benefícios aos indivíduos e a instituição, assim como contribui para o desenvolvimento e o diferencial competitivo organizacional. O compartilhamento de conhecimento nas organizações está relacionado à atitude dos indivíduos, aos contextos organizacionais e culturais, seja no nível local ou macroambiente, ao acesso e disseminação dos recursos tecnológicos de informação e comunicação, assim como "a cultura favorável, os fatores estruturais, motivacionais e humanos" (RIZZON et al., 2016)

O compartilhamento de conhecimento possibilita a produção de conhecimento e não apenas a aquisição de conhecimentos já produzidos. Na produção de conhecimento, diferentemente da aquisição de conhecimento, são valorizados a capacidade das pessoas de construir seu conhecimento, seu processo de aprendizagem, suas atitudes e suas habilidades (autonomia, autoavaliação contínua, criatividade, responsabilidade compartilhada). Nesta perspectiva, toda a aquisição de conhecimento produzido é passível de compartilhamento, contribuindo para a mediação da ação desse conhecimento que se supõe uma reconstrução por parte do indivíduo (MIRANDA, 1997).

A partir do estudo realizado, sugere-se, em pesquisas futuras, ampliar as bases de dados a serem consultadas, da mesma forma que as categorias e subcategorias de barreiras identificadas visando facilitar sua compreensão e inclusão de ações que possam mitigar ou até mesmo que possam superar as barreiras. Da mesma forma sugere-se a inclusão de estudos de caso, nos quais possam ser evidenciados os resultados dessas pesquisas.

\section{REFERÊNCIAS}

ALCARA, A. R. et al. Fatores que influenciam o compartilhamento da informação e do conhecimento. Perspectivas em Ciência da Informação, v. 14, n. 1, p. 170-191, jan/abr, 2009.

ALVARENGA NETO, R. C. D. Gestão do Conhecimento em Organizações: Proposta de Mapeamento Conceitual Integrativo. São Paulo: Saraiva, 2008.

AKGÜN, A. E.; KESKIN, H.; AYAR, H.; OKUNAKOL, Z. Knowledge sharing barriers in software development teams: a multiple case study in Turkey, Kybernetes, v. 46, n. 4, p. 603-620, 2017.

ASRAR-UL-HAQ, M.; ANWAR, S. A systematic review of knowledge management and knowledge sharing: Trends, issues, and challenges. Cogent Business \& Management, v. 3, n. 1, p. 1-17, 2016.

ATLAS.TI. Atlas.ti 7 for mac. Disponível em: <www.atlasti.com>. Acesso em: 20 jun. 2018.

BLOICE, L.; BURNETT, S. Barriers to knowledge sharing in third sector social care: a case study. Journal of Knowledge Management, v. 20, n. 1, p. 125-145, 2016.

CHOO, C. W. A organização do conhecimento: como as organizações usam a informação para criar significado, construir conhecimento e toma decisões. 2. ed. São Paulo: Editora Senac, 2006.

DAVENPORT, T. H.; PRUSAK, L. Conhecimento empresarial. Rio de Janeiro: Campus,1998

Perspectivas em Gestão \& Conhecimento, João Pessoa, v. 8, número especial, p. 86-101, out. 2018. 
DI CHIARA, I. G.; ALCARA, A. R.; TOMAEL, M. I. Tipos de compartilhamento de informação e conhecimento no ambiente de P\&D. Inf. \& Soc. Est., João Pessoa, v. 20, n. 2, p. 105-118, maio/ago, 2010.

DISTERER, G. Individual and social barriers to knowledge transfer. In: Proceedings of the 34th Hawaii international conference on system sciences, Hawaii, USA, January 3-6, 2001

GHOBADI, S.; MATHIASSEN, L. Perceived barriers to effective knowledge sharing in agile software teams. Information Systems Journal, p. 1-32, 2015.

GIDDENS, A. As conseqüências da modernidade. Tradução: Raul Fiker. 2a ed. São Paulo: Editora Unesp, 1991.

GIDER, Ö.; OCAK, S.; TOP, M. Perceptions of Physicians About Knowledge Sharing Barriers in Turkish Health Care System. Journal of Medical Systems, v. 49, n. 42, p. 1-13, 2015.

GIL, A. C. Métodos e Técnicas de Pesquisa Social. São Paulo: Atlas, 2008.

HONG, D.; SUH, E.; KOO, C. Developing strategies for overcoming barriers to knowledge sharing based on conversational knowledge management: A case study of a financial company. Expert systems with Applications, v. 38, n. 12, p. 14417-14427, 2011.

HOOFF, B.; HUYSMAN, M. Managing knowledge sharing: emergent and engineering approaches. Information \& Management, v. 46, p. 1-8, 2009.

KUKKO, M. Knowledge sharing barriers in organic growth: a case study from a software company. Journal of High Technology Management Research, v.24, p. 18-29, 2013.

LEE, C.; AUSTIN, M. J. Building organisational supports for knowledge sharing in county human service organisations: a cross-case analysis of works-in progress. Journal of Evidence-Based Social Work, v. 9, n. 1/2, p. 3-18, 2012.

MARONATO, E. L. S.; STANKOWITZ, R. F. Gestão do conhecimento: o elemento confiança para o compartilhamento do conhecimento. Gestão e Desenvolvimento em Revista, v.3, n.2, p. 112-126, jul./dez. 2017.

MIRANDA, M. G. Novo paradigma de conhecimento e políticas educacionais na América Latina. Cadernos de Pesquisa, n. 110, p.37-48, mar., 1997.

NONAKA, I.; TAKEUCHI, H. A criação do conhecimento na empresa: como as empresas japonesas geram a dinâmica da organização. Rio de Janeiro: Campus, 1997

NONAKA, I.; TAKEUCHI, H. Criação de conhecimento na empresa: como as empresas japonesas geram a dinâmica da inovação. Tradução: Ana Beatriz Rodrigues, Priscilla Martins Celeste. 11a. ed. Rio de Janeiro: Elsevier, 2003.

NONAKA, I.; TAKEUCHI, H. Gestão do conhecimento. Porto Alegre: Bookman, 2008.

NONAKA, I; TOYAMA, R; KONNO, N. SECl, Ba and Leadership: a Unified Model of Dynamic Knowledge Creation. Long Range Planning, v.33, n1, p. 5-34, 2000.

Perspectivas em Gestão \& Conhecimento, João Pessoa, v. 8, número especial, p. 86-101, out. 2018. 
OLANIRAN, O. J.Barriers to Tacit Knowledge Sharing in Geographically Dispersed Project Teams in Oil and Gas Projects, Project Management Journal, v. 48, n. 3, p. 41-57, 2017.

OLIVEIRA, M. et al. Proposta de um modelo de maturidade para Gestão do Conhecimento: $\mathrm{KM}^{3}$. Revista Portuguesa e Brasileira de Gestão, Lisboa, v. 10, n. 4, out. 2011.

ORDAZ, C.; CRUZ J.; GINEL E. Facilitadores de los procesos de compartir conocimiento y su influencia sobre la innovación, JEL Code: M1 y 03. Grupos de investigación SEC2006-15105 y SEJ-02478, 2009.

PATALAS-MALISZEWSKA, J. Knowledge sharing barriers in the Polish manufacturing companies, Journal of International Studies, v. 7, n. 1, p. 27-34, 2014.

PAULIN, D.; WINROTH, M. Facilitators, Inhibitors, and Obstacles-A Refined Categorization Regarding Barriers for Knowledge Transfer, Sharing, and Flow. In: Proceedings of the 10th International Conference on Intellectual Capital, knowledge Management and Organisational Learning: ICICKM 2013. Academic Conferences Limited, 2013. p. 320.

PEREIRA, S. L. C. S.; BARBOSA, E. C. Compartilhamento do conhecimento: uma ferramenta elementar para o bom desenvolvimento de uma empresa. Revista Eletrônica de Interesse Social, v.1, n.3, p.1-15, 2018.

PUPULIM, M. E. H; FIALHO, F. A. P.; VARVÁKIS, G. Barreiras culturais à efetivação da gestão do conhecimento nas organizações públicas: relato de pesquisa. Inf. \& Soc.: Est., João Pessoa, v. 27, n. 3, p. 219-240, set/dez. 2017.

QUANDT, C. O.; PEREIRA JR., P. R.; VALE, R. R. Capital social e conhecimento: como as relações interpessoais permitem a criação do conhecimento em uma empresa de desenvolvimento de software. In: KM BRASIL - KM BRASIL - CONGRESSO BRASILEIRO DE GESTÃO DO CONHECIMENTO, 12, 2014, São Paulo. Anais... São Paulo: Sociedade Brasileira de Gestão do Conhecimento, 2014, p. 1-16.

RIEGE, A. Thee-dozen knowledge-sharing barriers managers must consider. Journal of Knowledge Management, v. 9, n. 3, p. 18-35, 2005.

RIZZON F., TAMIOSSO L. C., FACHINELLI A. C. Barreiras ao Compartilhamento do Conhecimento nas Organizações: Revisão Sistemática de Literatura. XVI Mostra de Iniciação Científica, Pósgraduação, Pesquisa e Extensão. Programa de Pós-Graduação em Administração - UCS, 2016.

RUNTE, G. I. B. C. Gestão do conhecimento: os desafios da implantação de um modelo integrado: o caso ANS. 2011. 146 f. Dissertação (Mestrado em Administração Pública) Fundação Getúlio Vargas, Rio de Janeiro. 2016.

SANTIAGO, J. R. S. Gestão do Conhecimento - a chave para o Sucesso Empresarial, Editora Novatec, 2004.

SANTOS, R. F.; OLIVEIRA, M.; LUCIANO, E. M. Havia um tempo em que era preciso motivar o compartilhamento de conhecimento, será agora tempo de proteger? Revista de Administração IMED, Passo Fundo, v.8, n.1, p.216-236, jan./jun. 2018.

Perspectivas em Gestão \& Conhecimento, João Pessoa, v. 8, número especial, p. 86-101, out. 2018. 
SANTOS, V. R.; SOARES, A. L.; CARVALHO, J. Á. Knowledge sharing barriers in complex research and development projects: an exploratory study on the perceptions of project managers. Knowledge and Process Management, v. 19, n. 1, p. 27-38, 2012.

SCHLESINGER, C. C. B. et al. Gestão do conhecimento na Administração Pública. Curitiba: Instituto Municipal de Administração Pública - IMAP, 2008.

SHARMA, B. P.; SINGH, M. D.; NEHA. Knowledge Sharing Barriers: An Approach of Interpretive Structural Modeling. The IUP Journal of Knowledge Management, v. X, n. 3, 2012.

SOLLI-SAETHER, H.; KARLSEN, J. T. VAN OORSCHOT, K. Strategic and Cultural Misalignment: Knowledge Sharing Barriers in Project Networks. Project Management Journal, v. 46, n. 3, p. 49-60, 2015.

TERRA, J. C. C. \& GORDON, C. Gestão do Conhecimento: o grande desafio empresarial: uma abordagem baseada no aprendizado e na criatividade. São Paulo. 2001.

WILBERT, et al. Compartilhamento do conhecimento em uma empresa pública brasileira: os correios. In: KM BRASIL - CONGRESSO BRASILEIRO DE GESTÃO DO CONHECIMENTO, 12, 2014. São Paulo. Anais... São Paulo: Sociedade Brasileira de Gestão do Conhecimento, 2014.

ZAHEDI, M.; SHAHIN, M.; BABAR, M. A systematic review of knowledge sharing challenges and practices in global software development. International Journal of Information Management, v. 36, n. 6, p. 995-1019, 2016.

ZHOU, L.; NUNES, B. N. Barriers to knowledge sharing in Chinese healthcare referral services: an emergent theoretical model. Glob Health Action v. 9, 2016.

Artigo recebido em 11/10/2018 e aceito para publicação em 29/10/2018

Perspectivas em Gestão \& Conhecimento, João Pessoa, v. 8, número especial, p. 86-101, out. 2018. 